\title{
CENTRO-AFFINE CURVATURE FLOWS ON CENTRALLY SYMMETRIC CONVEX CURVES
}

\author{
MOHAMMAD N. IVAKI
}

\begin{abstract}
We consider two types of $p$-centro-affine flows on smooth, centrally symmetric, closed convex planar curves: $p$-contracting and $p$-expanding. Here $p$ is an arbitrary real number greater than 1 . We show that, under any $p$-contracting flow, the evolving curves shrink to a point in finite time and the only homothetic solutions of the flow are ellipses centered at the origin. Furthermore, the normalized curves with enclosed area $\pi$ converge, in the Hausdorff metric, to the unit circle modulo $S L(2)$. As a $p$-expanding flow is, in a certain way, dual to a contracting one, we prove that, under any $p$-expanding flow, curves expand to infinity in finite time, while the only homothetic solutions of the flow are ellipses centered at the origin. If the curves are normalized to enclose constant area $\pi$, they display the same asymptotic behavior as the first type flow and converge, in the Hausdorff metric, and up to $S L(2)$ transformations, to the unit circle. At the end of the paper, we present a new proof of the $p$-affine isoperimetric inequality, $p \geq 1$, for smooth, centrally symmetric convex bodies in $\mathbb{R}^{2}$.
\end{abstract}

\section{INTRODUCTION}

The affine normal flow is a widely recognized evolution equation for hypersurfaces in which each point moves with velocity given by the affine normal vector. This evolution equation is the simplest affine invariant flow in differential geometry and it arises naturally if one considers families of $\delta$-convex floating bodies of a convex body [5, 30]. On the applicability aspect, the affine normal flow appears in image processing as a fundamental smoothing tool [23,24,26]. It also provides a nice proof of the Santaló inequality for convex hypersurfaces and, respectively, for the classical affine isoperimetric inequality, both due to Andrews [2]. The affine normal evolution has also been implicitly deployed by Stancu in 29, 30 for a breakthrough towards the homothety conjecture for convex floating bodies by Schütt-Werner [28]. In this paper, we consider an extension of the affine normal flow, namely the $p$-centro-affine flow introduced by Stancu [31, and we investigate its behavior. The $p$-centro-affine flows are natural generalizations of the affine normal flow in a way which will be explained below.

Let $K$ be a compact, centrally symmetric, strictly convex body, smoothly embedded in $\mathbb{R}^{2}$. We denote the space of such convex bodies by $\mathcal{K}_{\text {sym }}$. Let

$$
x_{K}: \mathbb{S}^{1} \rightarrow \mathbb{R}^{2}
$$

be the Gauss parametrization of $\partial K$, the boundary of $K \in \mathcal{K}_{\text {sym }}$, where the origin of the plane is chosen to coincide with the center of symmetry of the body. The

Received by the editors September 17, 2011 and, in revised form, June 11, 2012.

2010 Mathematics Subject Classification. Primary 53C44, 53A04, 52A10, 53A15; Secondary $35 \mathrm{~K} 55$. 
support function of $\partial K$ is defined by

$$
s_{\partial K}(z):=\left\langle x_{K}(z), z\right\rangle
$$

for each $z \in \mathbb{S}^{1}$. We denote the curvature of $\partial K$ by $\kappa$ and, furthermore, the radius of curvature of the curve $\partial K$ by $\mathfrak{r}$, both as functions on the unit circle. The latter is related to the support function by

$$
\mathfrak{r}[s](z):=\frac{\partial^{2}}{\partial \theta^{2}} s(z)+s(z)
$$

where $\theta$ is the angle parameter on $\mathbb{S}^{1}$. Let $K_{0} \in \mathcal{K}_{\text {sym }}$. We consider a family $\left\{K_{t}\right\}_{t} \in \mathcal{K}_{\text {sym }}$ given by the smooth map $x: \mathbb{S}^{1} \times[0, T) \rightarrow \mathbb{R}^{2}$, which is evolving according to the $p$-centro-affine flow, namely,

$$
\frac{\partial}{\partial t} x:=-\left(\frac{\kappa}{s^{3}}\right)^{\frac{p}{p+2}-\frac{1}{3}} \kappa^{\frac{1}{3}} z, x(\cdot, 0)=x_{K_{0}}(\cdot)
$$

for a fixed $p \geq 1$.

The flow (1.1) without the assumption of symmetry was defined in 31, in all dimensions in the class of $C_{+}^{2}$ convex bodies having origin in their interiors, for the purpose of finding new global centro-affine invariants of smooth convex bodies. Stancu obtains many interesting isoperimetric type inequalities via short time existence of the flow. Furthermore, her $p$-flow approach led to a geometric interpretation of the $L_{\phi}$ surface area recently introduced by Ludwig and Reitzner [12. The case $p=1$, the well-known affine normal flow, was already addressed by Andrews 2 in arbitrary dimension, by Sapiro and Tannenbaum 25] for convex planar curves, and by Angenent, Sapiro, and Tannenbaum [6] for non-convex curves. In [25], it was proved that the flow evolves any initial strictly convex curve, not necessarily symmetric, until it shrinks to an elliptical point. Andrews, in [2, investigated completely affine normal flow of hypersurfaces of any dimension and showed that the normalized flow evolves any initial strictly convex hypersurface exponentially fast, in the $C^{\infty}$ topology, to an ellipsoid. He also proves, in [5], that any convex initial bounded open set shrinks to a point in finite time under the affine normal flow. In 6], the authors prove convergence to a point under the affine normal flow starting from any $C^{2}$ planar curve, not necessarily convex, despite the fact that affine differential geometry is not defined for non-convex curves or hypersurfaces. In another direction, interesting results for the affine normal flow have been obtained in [1] by Loftin and Tsui regarding ancient solutions, and existence and regularity of solutions on non-compact strictly convex hypersurfaces. It is necessary to point out that the case $p=1$, in contrast to the case $p>1$, is the only instance when the flow (1.1) is no longer anisotropic. Moreover, the main difference between $p=1$ and the other cases is that for $p>1$ the flow is sensitive to the origin (as the name $p$-centro affine suggests) meaning that Euclidean translations of an initial curve will lead to different solutions, since translations affect the support function of the convex body which appears in (1.1). The translation invariancy of a flow is a main ingredient to prove the convergence to a point $[1,3,5]$. We overcome these issues, and other difficulties in the study of the asymptotic behavior of this flow, by restricting it to $\mathcal{K}_{\text {sym }}$ and implementing the $p$-affine isoperimetric inequalities developed by Lutwak [13. This approach emphasizes the usefulness of the $p$-affine surface area and the $p$-affine isoperimetric inequalities which have also been successfully employed by 
Lutwak and Oliker in [14 for obtaining regularity of the solutions to a generalization of the Minkowski problem. See [7,15, 19, 32,33, for more applications of these invaluable tools.

We will now give a description of (1.1) in terms of $S L(2)$-invariant quantities. Although it relies on introducing new notation, it provides an alternate view in which (1.1) is naturally an affine invariant flow. Equation (1.1) is precisely

$$
\frac{\partial}{\partial t} x:=\kappa_{0}^{\frac{p}{p+2}} \mathfrak{n}_{0}
$$

where $\mathfrak{n}_{0}$ denotes the centro-affine normal vector field along $\partial K$, and, in every direction, $\kappa_{0}=\frac{\kappa}{s^{3}}$ is the centro-affine curvature along the boundary. The centroaffine normal vector field is, pointwise, a multiple of the affine normal vector field $\mathfrak{n}$ which is known to be transversal to the boundary of $K$, but not necessarily orthogonal to it. More precisely, $\mathfrak{n}_{0}=\kappa_{0}^{-\frac{1}{3}} \mathfrak{n}$. In this paper, we choose to work with the flow's definition as a time-dependent anisotropic flow by powers of the Euclidean curvature and we will resort to the affine differential setting only for a technical step in the study of the normalized evolution.

Finally, note that the solution of (1.1) remains in $\mathcal{K}_{\text {sym }}$, as $s$ and $\kappa$ are symmetric in the sense

$$
\forall \theta: s(\theta+\pi)=s(\theta), \kappa(\theta+\pi)=\kappa(\theta) .
$$

Here and hereafter, we identify $z=(\cos \theta, \sin \theta)$ with the normal angle $\theta$ itself. We will give a proof of the fact that $K_{t} \in \mathcal{K}_{\text {sym }}$ as long as the flow exists in Lemma 2.3 .

We can rewrite the evolution equation (1.1) as a scalar parabolic equation for the support functions on the unit circle:

$$
\begin{gathered}
s: \mathbb{S}^{1} \times[0, T) \rightarrow \mathbb{R}^{+} \\
\frac{\partial}{\partial t} s=-s^{1-\frac{3 p}{p+2}} \mathfrak{r}^{-\frac{p}{p+2}}, s(\cdot, 0)=s_{\partial K}(\cdot), s(\cdot, t)=s_{\partial K_{t}}(\cdot),
\end{gathered}
$$

leading, in general, to an anisotropic planar evolution. As in [3], it can be shown that there is a one-to-one correspondence between the solutions of (1.1) and those of (1.2).

The convex body $K^{\circ}$, dual to the convex body $K \in \mathcal{K}_{\text {sym }}$, is defined by

$$
K^{\circ}=\left\{y \in \mathbb{R}^{2} ;|\langle y, x\rangle| \leq 1 \text { for every } x \in K\right\} .
$$

In 31, the following expanding $p$-centro-affine flow was defined in connection to (1.1):

$$
\frac{\partial}{\partial t} x:=s\left(\frac{\kappa}{s^{3}}\right)^{-\frac{p}{p+2}} z, x(\cdot, 0)=x_{K_{0}^{\circ}}(\cdot) .
$$

It is easy to check as $K_{t}$ evolves according to (1.1), then $K_{t}^{\circ}$ evolves according to (1.3). Equivalently, the support function of $\partial K_{t}^{\circ}, s_{\partial K_{t}^{\circ}}$, evolves according to

$$
\frac{\partial}{\partial t} s=s^{1+\frac{3 p}{p+2} \mathfrak{r}^{\frac{p}{p+2}}}, \quad p \geq 1,
$$

with the initial condition $s(\cdot, 0)=s_{K_{0}^{\circ}}(\cdot)$; see Lemma 5.1

At a point $x$ of $\partial K$, the centro-affine curvature mentioned earlier is inversely proportional to the square of the area of the centered osculating ellipse at $x$. The centro-affine curvature is thus constant along ellipses centered at the origin which are, therefore, evolving homothetically by (1.2), respectively (1.4). Coupled with 
the fact that these flows increase the product $\operatorname{Area}(K) \cdot \operatorname{Area}\left(K^{\circ}\right)$, which is known to reach the maximum for ellipses centered at the origin (Santaló inequality), and the applications of $p$-flow stated above it was natural to investigate the asymptotic behavior of the flows which a priori suggests convergence to ellipses. While this was the first objective of the paper, in the process we obtained sharp affine isoperimetric type inequalities. The latter is related to the $p$-affine surface area introduced by Lutwak in 13 which has been the subject of intense research since then; see 12 for a recent, outreaching work which motivates even the present work. Finally, to the best of our knowledge, this is the first study of an anisotropic curvature flow with time-dependent weight. We regard as weight, as well as anisotropic factor, a power of the support function of the evolving body. In this paper we prove the following two theorems:

Theorem 1.1. Let $p>1$. Let $x_{K_{0}}: \mathbb{S}^{1} \rightarrow \mathbb{R}^{2}$ be a smooth, strictly convex parametrization of $K_{0} \in \mathcal{K}_{\text {sym }}$. Then there exists a unique solution $x: \mathbb{S}^{1} \times[0, T) \rightarrow$ $\mathbb{R}^{2}$ of equation (1.1) with the initial data $x_{K_{0}}$. The solution remains smooth and strictly convex on $[0, T)$ for a finite time $T$ and it converges to the origin of the plane. The rescaled curves given by $\sqrt{\frac{\pi}{A_{t}}} x(\theta, t)$ converge in the Hausdorff metric to the unit circle modulo $S L(2)$.

Theorem 1.2. Let $p>1$. Let $x_{K_{0}}: \mathbb{S}^{1} \rightarrow \mathbb{R}^{2}$ be a smooth, strictly convex parametrization of $K_{0} \in \mathcal{K}_{\text {sym }}$. Then there exists a unique solution $x: \mathbb{S}^{1} \times[0, T) \rightarrow$ $\mathbb{R}^{2}$ of equation (1.3) with the initial data $x_{K_{0}}$. The solution remains smooth and strictly convex on $[0, T)$ for a finite time $T$ and it expands in all directions to infinity. The rescaled curves given by $\sqrt{\frac{\pi}{A_{t}}} x(\theta, t)$ converge in the Hausdorff metric to the unit circle modulo $S L(2)$.

The paper is structured as follows. The next section focuses on the $p$-contracting affine flow. We show that the evolving curves shrink to a point in finite time. To study the convergence of solutions, we resort to affine differential geometry in the third section. In the third section, we will obtain sharp affine isoperimetric inequalities along the flow. In the fourth section, we obtain a crucial result about the constant asymptotic value of the centro-affine curvature of any solution. It is here where we conclude the convergence of solutions to a circle modulo $S L(2)$. In the fifth section, we present the relation between the contracting and the expanding flows. Consequently, we deduce an analogous asymptotic behavior for the $p$-expanding affine flow. Finally, in the last section, we present a new proof of the $p$-affine isoperimetric inequality, $p \geq 1$, for smooth, centrally symmetric convex bodies in $\mathbb{R}^{2}$.

\section{Convergence to A point And homothetic SOlutions}

This section is devoted to the contracting $p$-centro-affine curvature flow. In what follows, by evolving curves we mean the curves that enclose the evolving convex bodies in $\mathcal{K}_{\text {sym }}$. We start by recalling two results from [31] whose proofs are obtained by standard methods employed for geometric PDEs.

Proposition 2.1 (Short-term existence and uniqueness). Let $K_{0}$ be a convex body belonging to $\mathcal{K}_{\text {sym }}$ and let $p \geq 1$. Then there exists a time $T>0$ for which equation (1.1) has a unique solution starting from $K_{0}$. 
Proposition 2.2 (Containment principle). If $K_{\text {in }}$ and $K_{\text {out }}$ are the two convex bodies in $\mathcal{K}_{\text {sym }}$ such that $K_{\text {int }} \subset K_{\text {out }}$ and $p \geq 1$, then $K_{\text {in }}(t) \subseteq K_{\text {out }}(t)$ for as long as the solutions $K_{\text {in }}(t)$ and $K_{\text {out }}(t)$ of (1.1) (with given initial data $K_{\text {in }}(0)=K_{\text {in }}$, $\left.K_{\text {out }}(0)=K_{\text {out }}\right)$ exist in $\mathcal{K}_{\text {sym }}$.

Lemma 2.3. Let $\left\{K_{t}\right\}_{t}$ be a solution of (1.1) where $K_{0} \in \mathcal{K}_{\text {sym }}$. Then, $K_{t} \in \mathcal{K}_{\text {sym }}$ as long as the flow exists.

Proof. Note that both $-x(\cdot+\pi, t)$ and $x(\cdot, t)$ satisfy (1.1) with initial data $-x(\cdot+\pi, 0)$ and $x(\cdot, 0)$, respectively. At time $t=0$ we have $-x(\cdot+\pi, 0)=x(\cdot, 0)$. Therefore, by Proposition 2.1 we conclude that $-x(\cdot+\pi, t)=x(\cdot, t)$ as long as the flow exists.

The following evolution equations can be derived by a direct computation.

Lemma 2.4. Under the flow (1.2), one has

$$
\begin{gathered}
\frac{\partial}{\partial t} \mathfrak{r}=-\frac{\partial^{2}}{\partial \theta^{2}}\left(s^{1-\frac{3 p}{p+2}} \mathfrak{r}^{-\frac{p}{p+2}}\right)-s^{1-\frac{3 p}{p+2}} \mathfrak{r}^{-\frac{p}{p+2}}, \\
\frac{d}{d t} A(t)=-\Omega_{p}(t),
\end{gathered}
$$

where $A(t):=A\left(K_{t}\right)=\frac{1}{2} \int_{\mathbb{S}^{1}} \frac{s}{\kappa} d \theta$ is the area enclosed by the evolving curve, hence the area of $K_{t}$, and $\Omega_{p}(t):=\Omega_{p}\left(K_{t}\right)=\int_{\mathbb{S}^{1}} \frac{s}{\kappa}\left(\frac{\kappa}{s^{3}}\right)^{\frac{p}{p+2}} d \theta$ is the p-affine length of $\partial K_{t}$.

In trying to prove the convergence of the evolving curves to a point, the main obstacle was that, except for the case $p=1$, we could not find a uniform lower bound on the curvature of evolving curves. However, we could show, with several fruitful consequences, that there exists an entire family of increasing quantities related to the speed of the flow, $s^{1-\frac{3 p}{p+2}} \mathfrak{r}^{-\frac{p}{p+2}}$.

Proposition 2.5. For $1 \leq q \leq \frac{2 p}{p+1}$, or $q=0$, the flow (1.2) increases in time:

$$
\min _{\theta \in \mathbb{S}^{1}}\left(s^{q}\left(\frac{\kappa}{s^{3}}\right)^{\frac{p}{p+2}}\right)(\theta, t)
$$

Proof. Using the evolution equations (1.2) and (2.1), we obtain

$$
\begin{aligned}
\frac{\partial}{\partial t}\left(s^{q-\frac{3 p}{p+2}} \mathfrak{r}^{-\frac{p}{p+2}}\right) & =\left(\frac{\partial}{\partial t} s^{q-\frac{3 p}{p+2}}\right) \mathfrak{r}^{-\frac{p}{p+2}}+s^{q-\frac{3 p}{p+2}} \frac{\partial}{\partial t} \mathfrak{r}^{-\frac{p}{p+2}} \\
& =-\left(q-\frac{3 p}{p+2}\right) s^{q-\frac{3 p}{p+2}-1} \mathfrak{r}^{-\frac{p}{p+2}}\left(s^{1-\frac{3 p}{p+2}} \mathfrak{r}^{-\frac{p}{p+2}}\right) \\
& +\frac{p}{p+2} \mathfrak{r}^{-\frac{p}{p+2}-1} s^{q-\frac{3 p}{p+2}}\left[\left(s^{1-\frac{3 p}{p+2}} \mathfrak{r}^{-\frac{p}{p+2}}\right)_{\theta \theta}+s^{1-\frac{3 p}{p+2}} \mathfrak{r}^{-\frac{p}{p+2}}\right] \\
& =\left(\frac{3 p}{p+2}-q\right) s^{q-\frac{6 p}{p+2}} \mathfrak{r}^{-\frac{2 p}{p+2}}+\frac{p}{p+2} s^{q-\frac{6 p}{p+2}+1} \mathfrak{r}^{-\frac{2 p}{p+2}-1} \\
& +\frac{p}{p+2} \mathfrak{r}^{-\frac{p}{p+2}-1} s^{q-\frac{3 p}{p+2}}\left(s^{1-\frac{3 p}{p+2}} \mathfrak{r}^{-\frac{p}{p+2}}\right)_{\theta \theta} .
\end{aligned}
$$


To apply the maximum principle, we need to bound the right-hand side of (2.3) from below:

$$
\begin{aligned}
\left(s^{1-\frac{3 p}{p+2}} \mathfrak{r}^{-\frac{p}{p+2}}\right)_{\theta \theta} & =\left(s^{q-\frac{3 p}{p+2}} \mathfrak{r}^{-\frac{p}{p+2}} s^{1-q}\right)_{\theta \theta} \\
& =s^{1-q}\left(s^{q-\frac{3 p}{p+2}} \mathfrak{r}^{-\frac{p}{p+2}}\right)_{\theta \theta}+s^{q-\frac{3 p}{p+2}} \mathfrak{r}^{-\frac{p}{p+2}}\left(s^{1-q}\right)_{\theta \theta} \\
& +2\left(s^{1-q}\right)_{\theta}\left(s^{q-\frac{3 p}{p+2}} \mathfrak{r}^{-\frac{p}{p+2}}\right)_{\theta} .
\end{aligned}
$$

At the point where the minimum of $s^{q-\frac{3 p}{p+2}} \mathfrak{r}^{-\frac{p}{p+2}}$ occurs, we have

$$
\left(s^{q-\frac{3 p}{p+2}} \mathfrak{r}^{-\frac{p}{p+2}}\right)_{\theta \theta} \geq 0
$$

and

$$
\left(s^{q-\frac{3 p}{p+2}} \mathfrak{r}^{-\frac{p}{p+2}}\right)_{\theta}=0 .
$$

Therefore, by equation (2.4), we obtain that, at that point,

$$
\begin{aligned}
\left(s^{1-\frac{3 p}{p+2}} \mathfrak{r}^{-\frac{p}{p+2}}\right)_{\theta \theta} & \geq s^{q-\frac{3 p}{p+2}} \mathfrak{r}^{-\frac{p}{p+2}}\left(s^{1-q}\right)_{\theta \theta} \\
& =s^{q-\frac{3 p}{p+2}} \mathfrak{r}^{-\frac{p}{p+2}}\left[(1-q) s^{-q} s_{\theta \theta}-(1-q) q\left(s^{-1-q}\right) s_{\theta}^{2}\right] \\
& \geq s^{q-\frac{3 p}{p+2}} \mathfrak{r}^{-\frac{p}{p+2}}\left[(1-q) s^{-q} s_{\theta \theta}\right] \\
& =s^{q-\frac{3 p}{p+2}} \mathfrak{r}^{-\frac{p}{p+2}}\left[(1-q) s^{-q}(\mathfrak{r}-s)\right] \\
& =(1-q) s^{-\frac{3 p}{p+2}} \mathfrak{r}^{-\frac{p}{p+2}+1}-(1-q) s^{-\frac{3 p}{p+2}+1} \mathfrak{r}^{-\frac{p}{p+2}}
\end{aligned}
$$

where, to pass from the second to the third line, we assumed that either $q=0$ or $q \geq 1$. Combining (2.3), (2.4), and (2.5), at the point where the minimum of $s^{1-\frac{3 p}{p+2}} \mathfrak{r}^{-\frac{p}{p+2}}$ occurs, we have

$$
\begin{aligned}
\frac{\partial}{\partial t}\left(s^{q-\frac{3 p}{p+2}} \mathfrak{r}^{-\frac{p}{p+2}}\right) \geq & \left(\frac{3 p}{p+2}-q\right) s^{q-\frac{6 p}{p+2}} \mathfrak{r}^{-\frac{2 p}{p+2}}+\frac{p}{p+2} s^{q-\frac{6 p}{p+2}+1} \mathfrak{r}^{-\frac{2 p}{p+2}-1} \\
& +\frac{p(1-q)}{p+2} s^{q-\frac{6 p}{p+2}} \mathfrak{r}^{-\frac{2 p}{p+2}}-\frac{p(1-q)}{p+2} s^{q-\frac{6 p}{p+2}+1} \mathfrak{r}^{-\frac{2 p}{p+2}-1} \\
& =\left(\frac{3 p}{p+2}-q+\frac{p(1-q)}{p+2}\right) s^{q-\frac{6 p}{p+2}} \mathfrak{r}^{-\frac{2 p}{p+2}} \\
& +\frac{p q}{p+2} s^{q-\frac{6 p}{p+2}+1} \mathfrak{r}^{-\frac{2 p}{p+2}-1} .
\end{aligned}
$$

Since

$$
\frac{3 p}{p+2}-q+\frac{p(1-q)}{p+2}
$$

is non-negative for $q \leq \frac{2 p}{p+1}$, the claim follows.

Consequently, we have that

Corollary 2.6. Convexity of the evolving curves is preserved as long as the flow exists.

Proof. By Proposition 2.5, setting $q=0$, we have that, as long as the flow exists,

$$
\min _{\theta \in \mathbb{S}^{1}}\left(\frac{\kappa}{s^{3}}\right)(\theta, t) \geq \min _{\theta \in \mathbb{S}^{1}}\left(\frac{\kappa}{s^{3}}\right)(\theta, 0) .
$$


This inequality implies

$$
\kappa(\theta, t) \geq s^{3}(\theta, t) \min _{\theta \in \mathbb{S}^{1}}\left(\frac{\kappa}{s^{3}}\right)(\theta, 0)>0,
$$

which is precisely the claim of the corollary.

Following an idea from [34, we consider the evolution of the function $\frac{s^{1-\frac{3 p}{p+2}} \mathfrak{r}^{-\frac{p}{p+2}}}{s-\rho}$, for some appropriate $\rho$, to obtain an upper bound on the speed of the flow as long as the inradii of the evolving curves are uniformly bounded from below.

Lemma 2.7. If there exists an $r>0$ such that $s \geq r$ on $[0, T)$, then $\kappa$ is uniformly bounded from above on $[0, T)$.

Proof. Define $\Psi(x, t):=\frac{s^{1-\frac{3 p}{p+2} \mathfrak{r}^{-\frac{p}{p+2}}}}{s-\rho}$, where $\rho=\frac{1}{2} r$. For convenience, we set $\alpha:=1-\frac{3 p}{p+2}$ and $\beta:=-\frac{p}{p+2}$. At the point where the maximum of $\Psi$ occurs, we have

hence we obtain

$$
\Psi_{\theta}=0, \Psi_{\theta \theta} \leq 0
$$

$$
\left(s^{\alpha} \mathfrak{r}^{\beta}\right)_{\theta \theta}+s^{\alpha} \mathfrak{r}^{\beta} \leq-\frac{\rho s^{\alpha} \mathfrak{r}^{\beta}-s^{\alpha} \mathfrak{r}^{\beta+1}}{s-\rho}
$$

Calculating

$$
\frac{\partial \Psi}{\partial t}=\frac{s^{\alpha}}{s-\rho} \frac{\partial \mathfrak{r}^{\beta}}{\partial t}+\frac{\mathfrak{r}^{\beta}}{s-\rho} \frac{\partial s^{\alpha}}{\partial t}-\frac{s^{\alpha} \mathfrak{r}^{\beta}}{(s-\rho)^{2}} \frac{\partial s}{\partial t}
$$

and using equation (2.1) and inequality (2.6), we infer that, at the point where the maximum of $\Psi$ is reached, we have

$$
0 \leq \frac{\partial}{\partial t} \Psi \leq \frac{1}{s-\rho}\left[-\beta s^{\alpha} \mathfrak{r}^{\beta-1}\left(-\frac{\rho s^{\alpha} \mathfrak{r}^{\beta}-s^{\alpha} \mathfrak{r}^{\beta+1}}{s-\rho}\right)+\mathfrak{r}^{\beta} \frac{\partial}{\partial t} s^{\alpha}+\frac{\left(s^{2 \alpha} \mathfrak{r}^{2 \beta}\right)}{s-\rho}\right] .
$$

This last inequality gives

$$
\beta \rho \kappa-\beta-\alpha+\alpha \rho \frac{1}{s}+1 \geq 0 .
$$

Neglecting the non-positive term $\alpha \rho \frac{1}{s}$, we obtain

$$
\beta \rho \kappa-\beta-\alpha+1 \geq 0 .
$$

Note that $\alpha+\beta-1=-\frac{4 p}{p+2}$, therefore $0 \leq \kappa \leq \frac{4}{\rho}$, consequently implying the lemma.

Lemma 2.8. Let $T$ be the maximal time of existence of the solution to the flow (1.2) with a fixed initial body $K_{0} \in \mathcal{K}_{\text {sym. }}$. Then $T$ is finite and the area of $K_{t}$, $A(t)$, tends to zero as $t$ approaches $T$.

Proof. Suppose that $S_{0}$ is a circle which, at time zero, encloses $K_{0}$. It is clear that, by applying the $p$-flow to $S_{0}$, the evolving circles $S_{t}$ converge to a point in finite time. By Proposition 2.2 $K_{t}$ remains in the closure of $S_{t}$, therefore $T$ must be finite. Suppose now that $A(t)$ does not tend to zero. Then we must have $s \geq r$, for some $r>0$ on $[0, T)$. By Corollary 2.6 and Lemma 2.7. the curvature of the solution remains bounded on $[0, T)$ from below and above. Consequently the evolution equation (1.2) is uniformly parabolic on $[0, T)$, and bounds on higher 
derivatives of the support function follows by [10] and Schauder theory. Hence, we can extend the solution after time $T$, contradicting its definition.

Lemma 2.9. Assume $1 \leq l<2$. Then any solution of the flow (1.2) satisfies $\lim _{t \rightarrow T} \Omega_{l}(t)=0$.

Proof. From the $p$-affine isoperimetric inequality in $\mathbb{R}^{2}[13$, we have

$$
0 \leq \Omega_{l}^{2+l}(t) \leq 2^{2+l} \pi^{2 l} A^{2-l}(t)
$$

for any $l \geq 1$. Therefore, the result is a direct consequence of Lemma 2.8

Proposition 2.10. Let $L(t)$ be the length of $\partial K_{t}$ as $K_{t}$ is evolving under (1.2). If $p \geq 1$, then $\lim _{t \rightarrow T} L(t)=0$.

Proof. We first seek an $l$ with the following simultaneous properties:

(1) $1 \leq l<2$,

(2) $1 \leq \frac{p}{p+2} \frac{l+2}{l} \leq \frac{2 p}{p+1}$.

Note that, by Lemma 2.9. condition (1) implies $\lim _{t \rightarrow T} \Omega_{l}(t)=0$. Condition (2) implies that

is increasing. Indeed

$$
\min _{\theta \in \mathbb{S}^{1}}\left(s\left(\frac{\kappa}{s^{3}}\right)^{\frac{l}{l+2}}\right)(\theta, t)
$$

$$
\left(\min _{\theta \in \mathbb{S}^{1}} s\left(\frac{\kappa}{s^{3}}\right)^{\frac{l}{l+2}}(\theta, t)\right)^{\frac{p}{p+2} \frac{l+2}{l}}=\min _{\theta \in \mathbb{S}^{1}}\left(s^{\frac{p}{p+2} \frac{l+2}{l}}\left(\frac{\kappa}{s^{3}}\right)^{\frac{p}{p+2}}\right)(\theta, t),
$$

therefore the claim follows from Proposition 2.5

We now proceed to prove the existence of such an $l$. Solving $\frac{p}{p+2} \frac{l+2}{l} \leq \frac{2 p}{p+1}$ implies $l \geq \frac{2 p+2}{p+3}$. Let

$$
l:=\frac{2 p+2}{p+3}
$$

and note that it satisfies both conditions (1) and (2). We further remark that

$$
\min _{\theta \in \mathbb{S}^{1}}\left(s\left(\frac{\kappa}{s^{3}}\right)^{\frac{l}{2+l}}\right)(\theta, t) \int_{\mathbb{S}^{1}} \frac{1}{\kappa} d \theta \leq \Omega_{l}(t)=\int_{\mathbb{S}^{1}} \frac{s}{\kappa}\left(\frac{\kappa}{s^{3}}\right)^{\frac{l}{2+l}} d \theta .
$$

Thus, by taking the limit as $t \rightarrow T$ on both sides of inequality (2.7), we obtain

$$
\lim _{t \rightarrow T} L(t)=\lim _{t \rightarrow T} \int_{\mathbb{S}^{1}} \frac{1}{\kappa} d \theta=0 .
$$

Proposition 2.11. Centered ellipses are the only homothetic solutions to (1.2).

Proof. Denote $A^{\circ}(t):=A\left(K_{t}^{\circ}\right)$ and observe that $A(t) A^{\circ}(t)$ is scale-invariant. Therefore for homothetic solutions this area product remains constant along the flow. Moreover, Proposition 2.2 in [31] states, in a larger generality, that, as long as the flow exists, the $p$-affine flow does not decrease the area product $A(t) A^{\circ}(t)$ and it remains constant if and only if the evolving curves are ellipses centered at the origin. The result follows now from the existence of solutions until the extinction time of evolving convex bodies which are centrally symmetric with the center of symmetry placed at the origin.

Alternately, one can argue that having a homothetic solution to (1.2) is equivalent to $\frac{\kappa}{s^{3}}$ being constant along the boundary of $K_{t}$. Then Petty's lemma [21] shows that the latter is equivalent to $K_{t}$ being an ellipse centered at the origin. 


\section{Affine differential Setting}

In what follows, we work in the affine setting to obtain a sharp affine isoperimetric inequality along the $p$-flow, Theorem 3.3 . We will now recall several definitions from affine differential geometry. Let $\gamma: \mathbb{S}^{1} \rightarrow \mathbb{R}^{2}$ be an embedded strictly convex curve with the curve parameter $\theta$. Define $\mathfrak{g}(\theta):=\left[\gamma_{\theta}, \gamma_{\theta \theta}\right]^{1 / 3}$, where, for two vectors $u, v$ in $\mathbb{R}^{2},[u, v]$ denotes the determinant of the matrix with rows $u$ and $v$. The affine arc-length is then given by

$$
\mathfrak{s}(\theta):=\int_{0}^{\theta} \mathfrak{g}(\xi) d \xi .
$$

Furthermore, the affine tangent vector $\mathfrak{t}$, the affine normal vector $\mathfrak{n}$, and the affine curvature are defined, in this order, as follows:

$$
\mathfrak{t}:=\gamma_{\mathfrak{s}}, \mathfrak{n}:=\gamma_{\mathfrak{s i}}, \quad \mu:=\left[\gamma_{\mathfrak{s s}}, \gamma_{\mathfrak{s s g}}\right]
$$

In the affine coordinate $\mathfrak{s}$, the following relations hold:

$$
\begin{aligned}
{\left[\gamma_{\mathfrak{s}}, \gamma_{\mathfrak{s s}}\right] } & =1, \\
{\left[\gamma_{\mathfrak{s}}, \gamma_{\mathfrak{s s s}}\right] } & =0, \\
{\left[\gamma_{\mathfrak{s i s s}}, \gamma_{\mathfrak{s}}\right] } & =\mu .
\end{aligned}
$$

Moreover, it can be easily verified that $\kappa_{0}=\frac{\left[\gamma_{\theta}, \gamma_{\theta \theta}\right]}{\left[\gamma, \gamma_{\theta}\right]^{3}}=\frac{\left[\gamma_{\mathfrak{s}}, \gamma_{\mathfrak{s} s}\right]}{\left[\gamma, \gamma_{\mathfrak{s}}\right]^{3}}$. Since $\left[\gamma_{\mathfrak{s}}, \gamma_{\mathfrak{s s}}\right]=1$, we conclude that $\kappa_{0}=\frac{1}{\left[\gamma, \gamma_{\mathfrak{s}}\right]^{3}}$. The affine support function is defined by $\sigma:=\kappa_{0}^{-1 / 3}$; see [4,20].

Let $K_{0} \in \mathcal{K}_{\text {sym }}$. We consider a family $\left\{K_{t}\right\}_{t} \in \mathcal{K}_{\text {sym }}$ given by the smooth map $x: \mathbb{S}^{1} \times[0, T) \rightarrow \mathbb{R}^{2}$, which is evolving according to

$$
\frac{\partial}{\partial t} x:=\sigma^{1-\frac{3 p}{p+2}} \mathfrak{n}, x(\cdot, 0)=x_{K_{0}}(\cdot)
$$

for a fixed $p \geq 1$. Observe that up to diffeomorphisms the flow defined in (3.3) is equivalent to the flow defined by (1.1).

Lemma 3.1. Let $\gamma(t):=\partial K_{t}$ be the boundary of the convex body $K_{t}$ evolving under the flow (3.3). Then the following evolution equations hold:

(1) $\frac{\partial}{\partial t} \mathfrak{g}=-\frac{2}{3} \mathfrak{g} \sigma^{1-\frac{3 p}{p+2}} \mu+\frac{1}{3} \mathfrak{g}\left(\sigma^{1-\frac{3 p}{p+2}}\right)_{\mathfrak{s j}}$,

(2) $\frac{\partial}{\partial t} \mathfrak{t}=\left[-\frac{1}{3} \sigma^{1-\frac{3 p}{p+2}} \mu-\frac{1}{3}\left(\sigma^{1-\frac{3 p}{p+2}}\right)_{\mathfrak{s} \mathfrak{s}}\right]^{\mathfrak{s}} \mathfrak{t}+\left(\sigma^{1-\frac{3 p}{p+2}}\right)_{\mathfrak{s}} \mathfrak{n}$,

(3) $\frac{\partial}{\partial t} \sigma=\sigma^{1-\frac{3 p}{p+2}}\left[-\frac{4}{3}+\left(\frac{p}{p+2}+1\right)\left(1-\frac{3 p}{p+2}\right) \frac{\sigma_{\mathfrak{s}}^{2}}{\sigma}+\frac{p}{p+2} \sigma_{\mathfrak{s s}}\right]$,

(4) $\frac{d}{d t} \Omega_{p}(t)=\frac{2(p-2)}{p+2} \int_{\gamma} \sigma^{1-\frac{6 p}{p+2}} d \mathfrak{s}+\frac{18 p^{2}}{(p+2)^{3}} \int_{\gamma} \sigma^{-\frac{6 p}{p+2}} \sigma_{\mathfrak{s}}^{2} d \mathfrak{s}$.

Proof. To prove the lemma we will repeatedly use equations (3.2) without further mention:

$$
\frac{\partial}{\partial t} \mathfrak{g}^{3}=\frac{\partial}{\partial t}\left[\gamma_{\theta}, \gamma_{\theta \theta}\right]=\left[\frac{\partial}{\partial t} \gamma_{\theta}, \gamma_{\theta \theta}\right]+\left[\gamma_{\theta}, \frac{\partial}{\partial t} \gamma_{\theta \theta}\right]
$$


We have that

$$
\begin{aligned}
{\left[\frac{\partial}{\partial t} \gamma_{\theta}, \gamma_{\theta \theta}\right] } & =\left[\frac{\partial}{\partial \theta}\left(\sigma^{1-\frac{3 p}{p+2}} \gamma_{\mathfrak{s s}}\right), \gamma_{\theta \theta}\right] \\
& =\left[\mathfrak{g} \frac{\partial}{\partial \mathfrak{s}}\left(\sigma^{1-\frac{3 p}{p+2}} \gamma_{\mathfrak{s s}}\right), \gamma_{\theta \theta}\right] \\
& =\mathfrak{g}\left[\left(\sigma^{1-\frac{3 p}{p+2}}\right)_{\mathfrak{s}} \gamma_{\mathfrak{s s}}+\sigma^{1-\frac{3 p}{p+2}} \gamma_{\mathfrak{s s s}}, \gamma_{\theta \theta}\right]
\end{aligned}
$$

Since $\frac{\partial^{2}}{\partial \theta^{2}}=\mathfrak{g} \mathfrak{g}_{\mathfrak{s}} \frac{\partial}{\partial \mathfrak{s}}+\mathfrak{g}^{2} \frac{\partial^{2}}{\partial \mathfrak{s}^{2}}$, we further have $\gamma_{\theta \theta}=\mathfrak{g}^{2} \gamma_{\mathfrak{s s}}+\mathfrak{g}_{\mathfrak{s}} \gamma_{\mathfrak{s}}$ and, therefore,

$$
\begin{aligned}
{\left[\frac{\partial}{\partial t} \gamma_{\theta}, \gamma_{\theta \theta}\right] } & =\mathfrak{g}\left[\left(\sigma^{1-\frac{3 p}{p+2}}\right)_{\mathfrak{s}} \gamma_{\mathfrak{s s}}+\sigma^{1-\frac{3 p}{p+2}} \gamma_{\mathfrak{s s s}}, \mathfrak{g}^{2} \gamma_{\mathfrak{s s}}+\mathfrak{g g}_{\mathfrak{s}} \gamma_{\mathfrak{s}}\right] \\
& =-\mathfrak{g}^{2} \mathfrak{g}_{\mathfrak{s}}\left(\sigma^{1-\frac{3 p}{p+2}}\right)_{\mathfrak{s}}-\mathfrak{g}^{3} \sigma^{1-\frac{3 p}{p+2}} \mu .
\end{aligned}
$$

On the other hand, we have

$$
\begin{aligned}
{\left[\gamma_{\theta}, \frac{\partial}{\partial t} \gamma_{\theta \theta}\right] } & =\left[\mathfrak{g} \gamma_{\mathfrak{s}}, \frac{\partial^{2}}{\partial \theta^{2}}\left(\sigma^{1-\frac{3 p}{p+2}} \gamma_{\mathfrak{s s}}\right)\right] \\
& =\left[\mathfrak{g} \gamma_{\mathfrak{s}}, \mathfrak{g} \mathfrak{g}_{\mathfrak{s}} \frac{\partial}{\partial \mathfrak{s}}\left(\sigma^{1-\frac{3 p}{p+2}} \gamma_{\mathfrak{s s}}\right)+\mathfrak{g}^{2} \frac{\partial^{2}}{\partial \mathfrak{s}^{2}}\left(\sigma^{1-\frac{3 p}{p+2}} \gamma_{\mathfrak{s s}}\right)\right] \\
& =\mathfrak{g}^{2} \mathfrak{g}_{\mathfrak{s}}\left(\sigma^{1-\frac{3 p}{p+2}}\right)_{\mathfrak{s}}+\mathfrak{g}^{3}\left(\sigma^{1-\frac{3 p}{p+2}}\right)_{\mathfrak{s} \mathfrak{s}}-\mathfrak{g}^{3} \sigma^{1-\frac{3 p}{p+2}} \mu .
\end{aligned}
$$

Hence, we conclude that

$$
\frac{\partial}{\partial t} \mathfrak{g}^{3}=\mathfrak{g}^{3}\left(\sigma^{1-\frac{3 p}{p+2}}\right)_{\mathfrak{s i s}}-2 \mathfrak{g}^{3} \sigma^{1-\frac{3 p}{p+2}} \mu,
$$

which verifies our first claim.

To prove the second claim, we observe that

$$
\frac{\partial}{\partial t} \frac{\partial}{\partial \mathfrak{s}}=\frac{\partial}{\partial \mathfrak{s}} \frac{\partial}{\partial t}-\frac{1}{\mathfrak{g}} \frac{\partial \mathfrak{g}}{\partial t} \frac{\partial}{\partial \mathfrak{s}} .
$$

By (3.4), we get

$$
\begin{aligned}
\frac{\partial}{\partial t} \mathfrak{t} & =\frac{\partial}{\partial t} \frac{\partial}{\partial \mathfrak{s}} \gamma \\
& =\frac{\partial}{\partial \mathfrak{s}}\left(\sigma^{1-\frac{3 p}{p+2}} \gamma_{\mathfrak{s} \mathfrak{s}}\right)+\left(\frac{2}{3} \sigma^{1-\frac{3 p}{p+2}} \mu-\frac{1}{3}\left(\sigma^{1-\frac{3 p}{p+2}}\right)_{\mathfrak{s s}}\right) \mathfrak{t} \\
& =\left(\sigma^{1-\frac{3 p}{p+2}}\right)_{\mathfrak{s}} \mathfrak{n}+\sigma^{1-\frac{3 p}{p+2}} \gamma_{\mathfrak{s s s}}+\left(\frac{2}{3} \sigma^{1-\frac{3 p}{p+2}} \mu-\frac{1}{3}\left(\sigma^{1-\frac{3 p}{p+2}}\right)_{\mathfrak{s} \mathfrak{s}}\right) \mathfrak{t}
\end{aligned}
$$

We note that $\gamma_{\mathfrak{s s \mathfrak { s }}}=-\mu \gamma_{\mathfrak{s}}$ ending the proof of the second claim.

We now proceed to prove the third claim with

$$
\frac{\partial}{\partial t} \sigma=\frac{\partial}{\partial t}\left[\gamma, \gamma_{\mathfrak{s}}\right]=\left[\frac{\partial}{\partial t} \gamma, \gamma_{\mathfrak{s}}\right]+\left[\gamma, \frac{\partial}{\partial t} \gamma_{\mathfrak{s}}\right]
$$


By the evolution equation (3.3), the evolution equation for $\mathfrak{t}$, and the identities $\sigma=\left[\gamma, \gamma_{\mathfrak{s}}\right]$ and $\sigma_{\mathfrak{s}}=\left[\gamma, \gamma_{\mathfrak{s s}}\right]$, we get that

$$
\begin{aligned}
\frac{\partial}{\partial t} \sigma & =\left[\sigma^{1-\frac{3 p}{p+2}} \gamma_{\mathfrak{s s}}, \gamma_{\mathfrak{s}}\right]+\left[\gamma,\left(-\frac{1}{3} \sigma^{1-\frac{3 p}{p+2}} \mu-\frac{1}{3}\left(\sigma^{1-\frac{3 p}{p+2}}\right)_{\mathfrak{s s}}\right) \gamma_{\mathfrak{s}}+\left(\sigma^{1-\frac{3 p}{p+2}}\right)_{\mathfrak{s}} \gamma_{\mathfrak{s i s}}\right] \\
& =-\sigma^{1-\frac{3 p}{p+2}}-\frac{1}{3} \sigma^{2-\frac{3 p}{p+2}} \mu-\frac{1}{3}\left(\sigma^{1-\frac{3 p}{p+2}}\right)_{\mathfrak{s s}} \sigma+\left(\sigma^{1-\frac{3 p}{p+2}}\right)_{\mathfrak{s}} \sigma_{\mathfrak{s}} \\
& =-\sigma^{1-\frac{3 p}{p+2}}-\frac{1}{3} \sigma^{2-\frac{3 p}{p+2}} \mu+\left(-\frac{1}{3}+\frac{p}{p+2}\right) \sigma^{1-\frac{3 p}{p+2}} \sigma_{\mathfrak{s} \mathfrak{s}} \\
& +\left(1-\frac{3 p}{p+2}\right) \frac{p}{p+2} \sigma^{-\frac{3 p}{p+2}} \sigma_{\mathfrak{s}}^{2}+\left(\sigma^{1-\frac{3 p}{p+2}}\right)_{\mathfrak{s}} \sigma_{\mathfrak{s}} .
\end{aligned}
$$

Observe that $\sigma_{\mathfrak{s s}}+\sigma \mu=1$, and apply it to the second and third terms of the last sum to obtain

$$
\begin{aligned}
\frac{\partial}{\partial t} \sigma & =-\frac{4}{3} \sigma^{1-\frac{3 p}{p+2}}+\frac{p}{p+2} \sigma^{1-\frac{3 p}{p+2}} \sigma_{\mathfrak{s s}}+\frac{p}{p+2}\left(1-\frac{3 p}{p+2}\right) \sigma^{-\frac{3 p}{p+2}} \sigma_{\mathfrak{s}}^{2}+\left(\sigma^{1-\frac{3 p}{p+2}}\right)_{\mathfrak{s}} \sigma_{\mathfrak{s}} \\
& =-\frac{4}{3} \sigma^{1-\frac{3 p}{p+2}}+\left(\frac{p}{p+2}+1\right)\left(1-\frac{3 p}{p+2}\right) \sigma^{-\frac{3 p}{p+2}} \sigma_{\mathfrak{s}}^{2}+\frac{p}{p+2}\left(\sigma^{1-\frac{3 p}{p+2}}\right) \sigma_{\mathfrak{s} \mathfrak{s}},
\end{aligned}
$$

as claimed.

For the last claim of the lemma, consider

$$
\begin{aligned}
\frac{d}{d t} \Omega_{p}(t) & =\frac{\partial}{\partial t} \int_{\gamma} \sigma^{1-\frac{3 p}{p+2}} d \mathfrak{s} \\
& =\int_{\gamma} \frac{\partial}{\partial t}\left(\sigma^{1-\frac{3 p}{p+2}}\right) d \mathfrak{s}+\int_{\gamma} \sigma^{1-\frac{3 p}{p+2}} \frac{\partial}{\partial t} d \mathfrak{s} .
\end{aligned}
$$

Using part (3) of the lemma and integration by parts, we obtain

$$
\begin{aligned}
\int_{\gamma} \frac{\partial}{\partial t} & \left(\sigma^{1-\frac{3 p}{p+2}}\right) d \mathfrak{s} \\
= & \left(\frac{4 p}{p+2}-\frac{4}{3}\right) \int_{\gamma} \sigma^{1-\frac{6 p}{p+2}} d \mathfrak{s}+\left(\frac{p}{p+2}+1\right)\left(1-\frac{3 p}{p+2}\right)^{2} \int_{\gamma} \sigma^{-\frac{6 p}{p+2}} \sigma_{\mathfrak{s}}^{2} d \mathfrak{s} \\
& +\frac{p}{p+2}\left(1-\frac{3 p}{p+2}\right) \int_{\gamma} \sigma^{1-\frac{6 p}{p+2}} \sigma_{\mathfrak{s s}} d \mathfrak{s} \\
= & \left(\frac{4 p}{p+2}-\frac{4}{3}\right) \int_{\gamma} \sigma^{1-\frac{6 p}{p+2}} d \mathfrak{s}+\left(\frac{p}{p+2}+1\right)\left(1-\frac{3 p}{p+2}\right)^{2} \int_{\gamma} \sigma^{-\frac{6 p}{p+2}} \sigma_{\mathfrak{s}}^{2} d \mathfrak{s} \\
& +\frac{p}{p+2}\left(1-\frac{3 p}{p+2}\right)\left(\frac{6 p}{p+2}-1\right) \int_{\gamma} \sigma^{-\frac{6 p}{p+2}} \sigma_{\mathfrak{s}}^{2} d \mathfrak{s} .
\end{aligned}
$$

On the other hand, (3.1) gives

$$
d \mathfrak{s}=\mathfrak{g} d \theta
$$

thus, by part (1), we get

$$
\frac{\partial}{\partial t} d \mathfrak{s}=\left[-\frac{2}{3} \sigma^{1-\frac{3 p}{p+2}} \mu+\frac{1}{3}\left(\sigma^{1-\frac{3 p}{p+2}}\right)_{\mathfrak{s i}}\right] d \mathfrak{s} .
$$


This implies

$$
\begin{aligned}
\int_{\gamma} \sigma^{1-\frac{3 p}{p+2}} \frac{\partial}{\partial t} d \mathfrak{s} \\
\quad=\int_{\gamma} \sigma^{1-\frac{3 p}{p+2}}\left[-\frac{2}{3} \sigma^{1-\frac{3 p}{p+2}} \mu+\frac{1}{3}\left(\sigma^{1-\frac{3 p}{p+2}}\right)_{\mathfrak{s} \mathfrak{s}}\right] d \mathfrak{s} \\
=-\frac{2}{3} \int_{\gamma} \sigma^{1-\frac{6 p}{p+2}}\left(1-\sigma_{\mathfrak{s s}}\right) d \mathfrak{s}-\frac{1}{3}\left(1-\frac{3 p}{p+2}\right)^{2} \int_{\gamma} \sigma^{-\frac{6 p}{p+2}} \sigma_{\mathfrak{s}}^{2} d \mathfrak{s} \\
\quad=-\frac{2}{3} \int_{\gamma} \sigma^{1-\frac{6 p}{p+2}} d \mathfrak{s}+\left[\frac{2}{3}\left(\frac{6 p}{p+2}-1\right)-\frac{1}{3}\left(1-\frac{3 p}{p+2}\right)^{2}\right] \int_{\gamma} \sigma^{-\frac{6 p}{p+2}} \sigma_{\mathfrak{s}}^{2} d \mathfrak{s} .
\end{aligned}
$$

Setting

$$
\begin{aligned}
Q: & =\left(\frac{p}{p+2}+1\right)\left(1-\frac{3 p}{p+2}\right)^{2}-\frac{p}{p+2}\left(\frac{3 p}{p+2}-1\right)\left(\frac{6 p}{p+2}-1\right) \\
& +\frac{2}{3}\left(\frac{6 p}{p+2}-1\right)-\frac{1}{3}\left(1-\frac{3 p}{p+2}\right)^{2}=\frac{18 p^{2}}{(p+2)^{3}}
\end{aligned}
$$

and combining the above equations, we finally acquire that

$$
\frac{d}{d t} \Omega_{p}(t)=\frac{2(p-2)}{p+2} \int_{\gamma} \sigma^{1-\frac{6 p}{p+2}} d \mathfrak{s}+Q \int_{\gamma} \sigma^{-\frac{6 p}{p+2}} \sigma_{\mathfrak{s}}^{2} d \mathfrak{s} .
$$

Now, we proceed to strengthen inequality (3.6). Let $K$ and $L$ be two convex bodies with support functions $s$ and $h$, respectively. Then the mixed volume of $K$ and $L$ is defined by

$$
V[s, h]=\int_{\mathbb{S}^{1}} s \mathfrak{r}[h] d \theta=\int_{\mathbb{S}^{1}} h \mathfrak{r}[s] d \theta .
$$

By Minkowski's mixed volume inequality [27, we have

$$
V^{2}[h, s] \geq V[s, s] V[h, h] .
$$

More interestingly, inequality (3.5) still holds if $h$ is an arbitrary function in $C^{2}\left(\mathbb{S}^{1}\right)$. Indeed, assuming that $h$ is not the support function of some convex body, for a large positive constant $c$, the sum $h+c s$ is a support function and we obtain, due to the linearity of mixed volumes,

$$
0 \leq V^{2}[h+c s, s]-V[h+c s, h+c s] V[s, s]=V^{2}[h, s]-V[h, h] V[s, s] .
$$

The following proposition, stated here only for $n=2$, is proved in 31 for all dimensions. Using our method in this section, we prove a stronger version of the planar inequality in Theorem 3.3 .

Proposition 3.2. Let $p \geq 1$, as $K_{t}$ evolves under (1.2). Then we have

$$
\frac{d}{d t} \Omega_{p}(t) \geq \frac{p-2}{p+2} \frac{\Omega_{p}^{2}(t)}{A(t)},
$$

with equality if and only if $K_{t}$ is an origin centered ellipse.

Theorem 3.3. The following strong affine isoperimetric inequalities hold. If $1 \leq$ $p \leq 2$, then

$$
\frac{d}{d t} \Omega_{p}(t) \geq \frac{p-2}{p+2} \frac{\Omega_{p}^{2}}{A}+\frac{18(p-1) p^{2}}{(p+2)^{3}} \int_{\gamma} \sigma^{-\frac{6 p}{p+2}} \sigma_{\mathfrak{s}}^{2} d \mathfrak{s}
$$


while, if $p \geq 2$, we then have

$$
\frac{d}{d t} \Omega_{p}(t) \geq \frac{p-2}{p+2} \frac{\Omega_{p}^{2}}{A}+\frac{18 p^{2}}{(p+2)^{3}} \int_{\gamma} \sigma^{-\frac{6 p}{p+2}} \sigma_{\mathfrak{s}}^{2} d \mathfrak{s}
$$

Proof. To prove the second statement, we note that Hölder's inequality gives

$$
\int_{\gamma} \sigma^{1-\frac{6 p}{p+2}} d \mathfrak{s}=\int_{\mathbb{S}^{1}} \frac{s}{\kappa}\left(\frac{\kappa}{s^{3}}\right)^{\frac{2 p}{p+2}} d \theta \geq \frac{\Omega_{p}^{2}}{2 A}
$$

and, thus, part (4) of Lemma 3.1 implies the affine isoperimetric inequality for $p \geq 2$. We now proceed to prove the first inequality. By Minkowski's mixed volume inequality (3.5), we have

$$
V\left[s\left(\frac{\kappa}{s^{3}}\right)^{\frac{p}{p+2}}, s\left(\frac{\kappa}{s^{3}}\right)^{\frac{p}{p+2}}\right] \leq \frac{V\left[s\left(\frac{\kappa}{s^{3}}\right)^{\frac{p}{p+2}}, s\right]^{2}}{V[s, s]} .
$$

Note that the right-hand side of the inequality is precisely $\frac{\Omega_{p}^{2}}{2 A}$. Using the identity (see [4])

$$
\begin{aligned}
\frac{\mathfrak{r}\left[s\left(\frac{\kappa}{s^{3}}\right)^{\frac{p}{p+2}}\right]}{\mathfrak{r}[s]} & =\frac{s}{\kappa^{\frac{1}{3}}}\left(\frac{\kappa}{s^{3}}\right)^{\frac{p}{p+2}} \mu+\left(\frac{s}{\kappa^{\frac{1}{3}}}\left(\frac{\kappa}{s^{3}}\right)^{\frac{p}{p+2}}\right)_{\mathfrak{s s}} \\
& =\sigma^{1-\frac{3 p}{p+2}} \mu+\left(\sigma^{1-\frac{3 p}{p+2}}\right)_{\mathfrak{s j}},
\end{aligned}
$$

we can rewrite the left-hand side of (3.9) as follows:

$$
\begin{aligned}
V\left[s\left(\frac{\kappa}{s^{3}}\right)^{\frac{p}{p+2}}, s\left(\frac{\kappa}{s^{3}}\right)^{\frac{p}{p+2}}\right] & =\int_{\mathbb{S}^{1}} s\left(\frac{\kappa}{s^{3}}\right)^{\frac{p}{p+2}} \mathfrak{r}\left[s\left(\frac{\kappa}{s^{3}}\right)^{\frac{p}{p+2}}\right] d \theta \\
& =\int_{\gamma} \frac{s}{\kappa^{\frac{1}{3}}}\left(\frac{\kappa}{s^{3}}\right)^{\frac{p}{p+2}} \frac{\mathfrak{r}\left[s\left(\frac{\kappa}{s^{3}}\right)^{\frac{p}{p+2}}\right]}{\mathfrak{r}} d \mathfrak{s} \\
& =\int_{\gamma} \sigma^{1-\frac{3 p}{p+2}}\left(\sigma^{1-\frac{3 p}{p+2}} \mu+\left(\sigma^{1-\frac{3 p}{p+2}}\right)_{\mathfrak{s s}}\right) d \mathfrak{s} \\
& =\int_{\gamma} \sigma^{2-\frac{6 p}{p+2}} \mu d \mathfrak{s}-\left(1-\frac{3 p}{p+2}\right)^{2} \int_{\gamma} \sigma^{-\frac{6 p}{p+2}} \sigma_{\mathfrak{s}}^{2} d \mathfrak{s} .
\end{aligned}
$$

Hence, combining equation (3.10) and inequality (3.9), we conclude that

$$
\int_{\gamma} \sigma^{2-\frac{6 p}{p+2}} \mu d \mathfrak{s} \leq \frac{\Omega_{p}^{2}}{2 A}+\left(1-\frac{3 p}{p+2}\right)^{2} \int_{\gamma} \sigma^{-\frac{6 p}{p+2}} \sigma_{\mathfrak{s}}^{2} d \mathfrak{s} .
$$


Inequality (3.11) is a special case of the affine-geometric Wirtinger inequality 4 , Lemma 6]. To finish the proof note also that

$$
\begin{aligned}
\frac{d}{d t} \Omega_{p}(t)= & \frac{2(p-2)}{p+2} \int_{\gamma} \sigma^{1-\frac{6 p}{p+2}} d \mathfrak{s}+\frac{18 p^{2}}{(p+2)^{3}} \int_{\gamma} \sigma^{-\frac{6 p}{p+2}} \sigma_{\mathfrak{s}}^{2} d \mathfrak{s} \\
= & \frac{2(p-2)}{p+2} \int_{\gamma} \sigma^{2-\frac{6 p}{p+2}}\left(\frac{1}{\sigma}-\frac{\sigma_{\mathfrak{s s}}}{\sigma}\right) d \mathfrak{s} \\
& +\frac{2(p-2)}{p+2} \int_{\gamma} \sigma^{2-\frac{6 p}{p+2}} \frac{\sigma_{\mathfrak{s s}}}{\sigma} d \mathfrak{s}+\frac{18 p^{2}}{(p+2)^{3}} \int_{\gamma} \sigma^{-\frac{6 p}{p+2}} \sigma_{\mathfrak{s}}^{2} d \mathfrak{s} \\
= & \frac{2(p-2)}{p+2} \int_{\gamma} \sigma^{2-\frac{6 p}{p+2}} \mu d \mathfrak{s} \\
& +\left[\frac{2(p-2)}{p+2}\left(\frac{6 p}{p+2}-1\right)+\frac{18 p^{2}}{(p+2)^{3}}\right] \int_{\gamma} \sigma^{-\frac{6 p}{p+2}} \sigma_{\mathfrak{s}}^{2} d \mathfrak{s},
\end{aligned}
$$

which, by inequality (3.11), implies

$$
\begin{aligned}
\frac{d}{d t} \Omega_{p}(t) \geq \frac{p-2}{p+2} \frac{\Omega_{p}^{2}}{A}+\left[\frac{2(p-2)}{p+2}\left(1-\frac{3 p}{p+2}\right)^{2}\right. \\
\left.\quad+\frac{2(p-2)}{p+2}\left(\frac{6 p}{p+2}-1\right)+\frac{18 p^{2}}{(p+2)^{3}}\right] \int_{\gamma} \sigma^{-\frac{6 p}{p+2}} \sigma_{\mathfrak{s}}^{2} d \mathfrak{s} \\
=\frac{p-2}{p+2} \frac{\Omega_{p}^{2}}{A}+\frac{18(p-1) p^{2}}{(p+2)^{3}} \int_{\gamma} \sigma^{-\frac{6 p}{p+2}} \sigma_{\mathfrak{s}}^{2} d \mathfrak{s} .
\end{aligned}
$$

Lemma 3.4. The p-affine isoperimetric ratio, $\frac{\Omega_{p}^{2+p}(t)}{A^{2-p}(t)}$, is non-decreasing along the flow (1.2) and remains constant if and only if $K_{t}$ is an origin centered ellipse.

Proof.

$$
\begin{aligned}
\frac{d}{d t} \frac{\Omega_{p}^{2+p}(t)}{A^{2-p}(t)} & =\frac{(2+p) \Omega_{p}^{p+1}(t) A^{2-p}(t) \frac{d}{d t} \Omega_{p}(t)+(2-p) A^{1-p}(t) \Omega_{p}^{3+p}(t)}{A^{2(2-p)}(t)} \\
& =\frac{\Omega_{p}^{1+p}(t)}{A^{2-p}(t)}\left((2+p) \frac{d}{d t} \Omega_{p}(t)-(p-2) \frac{\Omega_{p}^{2}(t)}{A(t)}\right) \geq 0,
\end{aligned}
$$

where we used inequality (3.6) on the last line.

Corollary 3.5. If $K_{t}$ evolves by (1.2) with extinction time $T$, the following limit holds:

$$
\lim \inf _{t \rightarrow T} \frac{\Omega_{p}^{p}(t)}{A^{1-p}(t)}\left[(2+p) \frac{d}{d t} \Omega_{p}(t)-(p-2) \frac{\Omega_{p}^{2}(t)}{A(t)}\right]=0 .
$$

Proof. By equations (2.2) and (3.12)

$$
\frac{d}{d t} \frac{\Omega_{p}^{2+p}(t)}{A^{2-p}(t)}=-\frac{d}{d t} \ln (A(t))\left(\frac{\Omega_{p}^{p}(t)}{A^{1-p}(t)}\left[(2+p) \frac{d}{d t} \Omega_{p}(t)-(p-2) \frac{\Omega_{p}^{2}(t)}{A(t)}\right]\right) .
$$

If

$$
\frac{\Omega_{p}^{p}(t)}{A^{1-p}(t)}\left[(2+p) \frac{d}{d t} \Omega_{p}(t)-(p-2) \frac{\Omega_{p}^{2}(t)}{A(t)}\right] \geq \varepsilon
$$


in a neighborhood of $T$, then

$$
\frac{d}{d t} \frac{\Omega_{p}^{2+p}(t)}{A^{2-p}(t)} \geq-\varepsilon \frac{d}{d t} \ln (A(t))
$$

Thus,

$$
\frac{\Omega_{p}^{2+p}}{A^{2-p}}(t) \geq \frac{\Omega_{p}^{2+p}}{A^{2-p}}\left(t_{1}\right)+\varepsilon \ln \left(A\left(t_{1}\right)\right)-\varepsilon \ln (A(t)),
$$

where the right-hand side goes to infinity as $A(t)$ goes to zero. This contradicts that the left-hand side is bounded from above by the $p$-affine isoperimetric inequality.

\section{Normalized FLOW}

In this section we study the normalized flows corresponding to the evolution described by (1.2). We consider the conventional rescaling such that the area enclosed by the normalized curves is $\pi$ by taking

$$
\tilde{s}_{t}:=\sqrt{\frac{\pi}{A(t)}} s_{t}, \tilde{\kappa}_{t}:=\sqrt{\frac{A(t)}{\pi}} \kappa_{t} .
$$

One can also define a new time parameter

$$
\tau=\int_{0}^{t}\left(\frac{\pi}{A\left(K_{t}\right)(\xi)}\right)^{\frac{2 p}{p+2}} d \xi
$$

and can easily verify that

$$
\frac{\partial}{\partial \tau} \tilde{s}=-\tilde{s}\left(\frac{\tilde{\kappa}}{\tilde{s}^{3}}\right)^{\frac{p}{p+2}}+\frac{\tilde{s}}{2 \pi} \tilde{\Omega}_{p}
$$

where $\tilde{\Omega}_{p}$ stands for the $p$-affine length of $\partial \tilde{K}_{t}$ having support function $\tilde{s}_{t}$. More precisely,

$$
\tilde{\Omega}_{p}(\tau):=\Omega_{p}\left(\tilde{K}_{\tau}\right)=\int_{\mathbb{S}^{1}} \frac{\tilde{s}}{\tilde{\kappa}}\left(\frac{\tilde{\kappa}}{\tilde{s}^{3}}\right)^{\frac{p}{p+2}} d \theta .
$$

However, even in the normalized case, we prefer to work on the finite time interval $[0, T)$.

Corollary 4.1. Let $p>1$, and let $\left\{t_{k}\right\}_{k}$ be the sequence of times realizing the limit (3.13) in Corollary 3.5. Then along the normalized contracting p-flow, we have

$$
\lim _{t_{k} \rightarrow T} \tilde{\sigma}\left(t_{k}\right)=1
$$

Proof. Since

$$
\frac{1}{\left(\frac{3 p}{p+2}-1\right)^{2}} \int_{\gamma}\left(\sigma^{1-\frac{3 p}{p+2}}\right)_{\mathfrak{s}}^{2} d \mathfrak{s}=\int_{\gamma} \sigma^{-\frac{6 p}{p+2}} \sigma_{\mathfrak{s}}^{2} d \mathfrak{s},
$$

by Theorem 3.3 and Corollary 3.5. we have

$$
\begin{aligned}
& 0=\lim _{t_{k} \rightarrow T} \frac{\Omega_{p}^{p}}{A^{1-p}}\left[\frac{d}{d t} \Omega_{p}(t)-\frac{p-2}{p+2} \frac{\Omega_{p}^{2}}{A}\right] \geq \lim _{t_{k} \rightarrow T} \frac{\Omega_{p}^{p}}{A^{1-p}}\left(\phi(p) \int_{\gamma}\left(\sigma^{1-\frac{3 p}{p+2}}\right)_{\mathfrak{s}}^{2} d \mathfrak{s}\right) \geq 0, \\
& \text { where } \phi(p):= \begin{cases}\frac{9 p^{2}}{2(p+2)(p-1)}, & \text { if } 1<p \leq 2, \\
\frac{9 p^{2}}{2(p+2)(p-1)^{2}}, & \text { if } p \geq 2 .\end{cases}
\end{aligned}
$$


As, by Lemma 3.4 the $p$-affine length $\tilde{\Omega}_{p}$ is increasing along the normalized flow, we conclude that, for any $p>1$,

$$
\lim _{t_{k} \rightarrow T} \int_{\tilde{\gamma}}\left(\tilde{\sigma}^{1-\frac{3 p}{p+2}}\right)_{\tilde{\mathfrak{s}}}^{2} d \tilde{\mathfrak{s}}=0 .
$$

We note that, for any $\theta_{1}, \theta_{2} \in \mathbb{S}^{1}$,

$$
\begin{aligned}
& \left|\int_{\theta_{1}}^{\theta_{2}}\left(\tilde{\sigma}^{1-\frac{3 p}{p+2}}\right)_{\theta} d \theta\right| \\
\leq & \int_{\mathbb{S}^{1}}\left|\left(\tilde{\sigma}^{1-\frac{3 p}{p+2}}\right)_{\theta}\right| d \theta=\int_{\tilde{\gamma}}\left|\left(\tilde{\sigma}^{1-\frac{3 p}{p+2}}\right)_{\tilde{\mathfrak{s}}}\right| d \tilde{\mathfrak{s}} \leq\left(\int_{\tilde{\gamma}}\left(\tilde{\sigma}^{1-\frac{3 p}{p+2}}\right)_{\tilde{\mathfrak{s}}}^{2} d \tilde{\mathfrak{s}}\right)^{1 / 2} \tilde{\Omega}_{1}^{1 / 2} .
\end{aligned}
$$

Take $\theta_{1}$ and $\theta_{2}$ to be two points where $\tilde{\sigma}$ reaches its extremal values. It is known that, for a smooth, simple curve with enclosed area $\pi, \min _{\mathbb{S}^{1}} \sigma \leq 1$ and $\max _{\mathbb{S}^{1}} \sigma \geq 1$; see Lemma 10 in 4 . Hence, as $\tilde{\Omega}_{1}$ is bounded from above by the classical affine isoperimetric inequality [13], we infer that $\lim _{t_{k} \rightarrow T} \tilde{\sigma}\left(t_{k}\right)=1$.

Lemma $4.2([8])$. Suppose that $K$ is a convex body in $\mathcal{K}_{\text {sym }}$. Denote the curvature and the support function of $\partial K$ respectively by $\kappa$ and $s$. If, for all $\theta: m \leq \frac{\kappa}{s^{3}}(\theta) \leq M$ for some positive numbers $m$ and $M$, then there exist two ellipses $\mathcal{E}_{\text {in }}$ and $\mathcal{E}_{\text {out }}$ such that $\mathcal{E}_{\text {in }} \subseteq K \subseteq \mathcal{E}_{\text {out }}$ and

$$
\frac{\kappa}{s^{3}}\left(\mathcal{E}_{\text {in }}\right)=M, \frac{\kappa}{s^{3}}\left(\mathcal{E}_{\text {out }}\right)=m .
$$

Proof. We present here the argument for the inner ellipse, the case of the outer one being similar. Recall that

$$
\kappa_{0}=\frac{[\dot{\gamma}, \ddot{\gamma}]}{[\gamma, \dot{\gamma}]^{3}},
$$

where $t \mapsto \gamma(t)$ is any counter-clockwise parametrization of the boundary curve. For an ellipse, this is a constant inverse proportional to the square of its area. So, we have to prove that the maximum-area ellipse contained in $K$ has $\kappa_{0} \leq M$. Let $\mathcal{E}_{i n}$ be the maximum-area ellipse contained in $K$. Since the problem is centro-affine invariant, we may assume that $\mathcal{E}_{\text {in }}$ is the unit circle. We will prove that $M \geq 1$. The result will then follow by shrinking the circle $\mathcal{E}_{\text {in }}$ until its centro-affine curvature is exactly $M$ and re-denoting it, for simplicity, the same way.

Consider the points where $\partial K$ touch $\mathcal{E}_{i n}$; one easily sees that there are at least four intersection points between $\partial K$ and $\mathcal{E}_{i n}$, otherwise $\mathcal{E}_{\text {in }}$ could be made larger. Thus, at least two of the intervals on the circle corresponding to the polar angle of the intersection points are not greater than $\pi / 2$. In fact, due to the symmetry of $K$, there exist at least two diametrically opposite such intervals. Choose coordinates so that one of the intersection points is $(1,0)$ and another intersection point is of the form $(\cos \theta, \sin \theta)$ for some $0<\theta \leq \pi / 2$. Observe that the arc of $\partial K$ between these touch points is contained in the square $[0,1] \times[0,1]$.

Parameterize $\partial K$ by the spanned area, i.e., by a curve $p \mapsto \gamma(p)$ such that $[\gamma, \dot{\gamma}]=1$. Therefore we have $[\gamma, \ddot{\gamma}]=0$, hence $\ddot{\gamma}(p)=-\kappa_{0}(p) \gamma(p)$, for all $p$, where $\kappa_{0}(p)$ is precisely the centro-affine curvature along the boundary of $K$. Let $\gamma(p)=(x(p), y(p))$; then $\ddot{x}(p)=-\kappa_{0}(p) x(p)$ and $\ddot{y}(p)=-\kappa_{0}(p) y(p)$. Suppose that $M=\sup \kappa_{0}(p)<1$. Since $x(0)=1, \dot{x}(0)=0, y(0)=0$, and $\dot{y}(0)=1$, a standard comparison theorem for equations of the form $\ddot{x}=-a^{2} x$ implies that $x(p)>\cos p$ and $y(p)>\sin p$ for all $p \in(0, \pi / 2]$. Therefore, $x(p)^{2}+y(p)^{2}>1$ for all $p \in(0, \pi / 2]$. 
This means that $\gamma$ leaves the square $[0,1] \times[0,1]$ before it has a chance to touch the circle again, contradicting our assumption.

Theorem 4.3. Suppose that $\tilde{s}_{t}$ is a solution of the normalized flow (4.1) for some initial convex body in $\mathcal{K}_{\text {sym }}$ and that $\left\{t_{k}\right\}_{k}$ is the sequence of times realizing the limit (3.13) in Corollary 3.5. Then there exist two families of centered ellipses $\left\{\mathcal{E}_{\text {in }}\left(t_{k}\right)\right\}$ and $\left\{\mathcal{E}_{\text {out }}\left(t_{k}\right)\right\}$ such that

$$
\mathcal{E}_{\text {in }}\left(t_{k}\right) \subseteq \tilde{K}_{t_{k}} \subseteq \mathcal{E}_{\text {out }}\left(t_{k}\right)
$$

Furthermore, the sequence of curves $\partial \tilde{K}_{t_{k}}$ converge, in the Hausdorff metric, to the unit circle modulo $S L(2)$.

Proof. By Corollary 4.1, we have

$$
\lim _{t_{k} \rightarrow T}\left(\frac{\tilde{\kappa}}{\tilde{s}^{3}}\right)\left(\theta, t_{k}\right)=1
$$

Thus, the first half of the claim follows from Lemma 4.2

Now we proceed to prove the second half of the claim. Evidently we can find an appropriate family of special linear transformations $\left\{L_{t_{k}}\right\}_{t_{k}}$ such that $L_{t_{k}}\left(\mathcal{E}_{\text {out }}\left(t_{k}\right)\right)$ is a circle at each time $t_{k}$. Each such area preserving linear transformation $L_{t_{k}}$ minimizes the Euclidean length of the ellipse $\mathcal{E}_{\text {out }}\left(t_{k}\right)$ at time $t_{k}$.

Thus, the construction of $\mathcal{E}_{\text {out }}\left(t_{k}\right), \mathcal{E}_{\text {in }}\left(t_{k}\right)$ implies

$$
\lim _{t_{k} \rightarrow T} L_{t_{k}}\left(\partial \mathcal{E}_{\text {out }}\left(t_{k}\right)\right)=\lim _{t_{k} \rightarrow T} L_{t_{k}}\left(\partial \mathcal{E}_{\text {in }}\left(t_{k}\right)\right)=\mathbb{S}^{1}
$$

in the Hausdorff metric.

Recall, from Lemma 4.2, that

$$
\min _{\theta \in \mathbb{S}^{1}}\left(\frac{\tilde{\kappa}}{\tilde{s}^{3}}\right)\left(\theta, t_{k}\right)=\frac{\kappa}{s^{3}}\left(\mathcal{E}_{\text {out }}\left(t_{k}\right)\right) .
$$

Since $\frac{\kappa}{s^{3}}$ is invariant under $S L(2)$, we have $\frac{\kappa}{s^{3}}\left(\mathcal{E}_{\text {out }}\left(t_{k}\right)\right)=\frac{\kappa}{s^{3}}\left(L_{t_{k}}\left(\mathcal{E}_{\text {out }}\left(t_{k}\right)\right)\right)$, therefore $\lim _{t_{k} \rightarrow T} \frac{\kappa}{s^{3}}\left(L_{t_{k}}\left(\mathcal{E}_{\text {out }}\left(t_{k}\right)\right)\right)=1$. This implies $\lim _{t_{k} \rightarrow T} L_{t_{k}}\left(\partial \mathcal{E}_{\text {out }}\left(t_{k}\right)\right)=\mathbb{S}^{1}$ in the Hausdorff metric. Similarly, from the choice of $\mathcal{E}_{i n}\left(t_{k}\right)$ in Lemma 4.2, we have that

$$
\max _{\theta \in \mathbb{S}^{1}}\left(\frac{\tilde{\kappa}}{\tilde{s}^{3}}\right)\left(\theta, t_{k}\right)=\frac{\kappa}{s^{3}}\left(\mathcal{E}_{i n}\left(t_{k}\right)\right),
$$

therefore $\lim _{t_{k} \rightarrow T} \frac{\kappa}{s^{3}}\left(L_{t_{k}}\left(\mathcal{E}_{i n}\left(t_{k}\right)\right)\right)=1$. This implies $\lim _{t_{k} \rightarrow T} A\left(L_{t_{k}}\left(\mathcal{E}_{i n}\left(t_{k}\right)\right)\right)=\pi$. As $L_{t_{k}}\left(\mathcal{E}_{\text {in }}\left(t_{k}\right)\right) \subseteq L_{t_{k}}\left(\mathcal{E}_{\text {out }}\left(t_{k}\right)\right)$, we conclude that $\lim _{t_{k} \rightarrow T} L_{t_{k}}\left(\partial \mathcal{E}_{\text {in }}\left(t_{k}\right)\right)=\mathbb{S}^{1}$ in the Hausdorff metric.

Now, applying $\left\{L_{t_{k}}\right\}_{t_{k}}$ to the inclusions (4.2), we obtain that $L_{t_{k}}\left(\tilde{K}_{t_{k}}\right)$ converges to the unit disk in the Hausdorff metric.

Corollary 4.4. Along the flow (4.1) with an arbitrary initial condition in $\mathcal{K}_{\text {sym }}$, we have

$$
\lim _{t \rightarrow T} A\left(K_{t}\right) A\left(K_{t}^{\circ}\right)=\pi^{2}
$$

for $p>1$.

Proof. Recall that the area product $A\left(K_{t}\right) A\left(K_{t}^{\circ}\right)$ is invariant under the general linear group, $G L(2)$, and increasing along any $p$-flow, unless the boundaries of the evolving convex bodies are centered ellipses. Moreover, as the convex bodies are centrally symmetric with the center of symmetry at the origin, the Santaló 
inequality gives $A(t) A^{\circ}(t) \leq \pi^{2}$ with equality if and only if the boundary curves are ellipses centered at the origin [22. Consequently, Theorem 4.3 implies the claim.

Before stating our main theorems let us recall the following frequently used fact in both convex geometry and analysis of PDEs which is due to Fritz John 1948.

Theorem 4.5 (John's Inclusion [9]). Suppose $K$ is a convex body in $\mathbb{R}^{n}$. Then there is a unique ellipse $\mathcal{E}_{J}$ of maximal volume contained in $K$. Furthermore, if $K$ is origin symmetric, then

$$
\mathcal{E}_{J} \subseteq K \subseteq \sqrt{n} \mathcal{E}_{J}
$$

Theorem 4.5 immediately implies if $K$ is an origin symmetric convex body whose volume is $\omega_{n}$, the volume of the unit ball in $\mathbb{R}^{n}$, then there is an affine transformation $L$ such that $r^{+}(L K) \leq \sqrt{n}$ and $r_{-}(L K) \geq \frac{1}{\sqrt{n}}$, where $r^{+}(L K)$ and $r_{-}(L K)$ are the inner radius and outer radius of $L K$ respectively. Now, we are ready to prove one of the main theorems:

Theorem 4.6. Let $p>1$. Suppose $\tilde{K}_{t}$ is a solution of the normalized flow (4.1) for some initial convex body in $\mathcal{K}_{\text {sym }}$. Then there exists a family of special linear transformations $\left\{L_{t}\right\}_{t \in[0, T)} \subset S L(2)$ such that $L_{t}\left(\partial \tilde{K}_{t}\right)$ converges to $\mathbb{S}^{1}$ in the Hausdorff metric.

Proof. At each time $t$, we apply a special linear transformation $L_{t}$ such that the Euclidean length of $\partial \tilde{K}_{t}$ is minimized. Let $\left\{t_{i}\right\}_{i}$ be a sequence of times converging to $T$. John's Inclusion or Proposition 8 of 4 implies the compactness of the set of convex bodies $L_{t_{i}}\left(\tilde{K}_{t_{i}}\right)$. By Corollary 4.4 and the Blaschke Selection Theorem, each subsequence of $L_{t_{i}}\left(\partial \tilde{K}_{t_{i}}\right)$ has a subsequence $L_{t_{i_{j}}}\left(\partial \tilde{K}_{t_{i_{j}}}\right)$ such that $L_{t_{i_{j}}}\left(\partial \tilde{K}_{t_{i_{j}}}\right)$ converges, in the Hausdorff metric, to an ellipse of enclosed area $\pi$. Thus, the length minimization condition rules out the degeneracy of the limit ellipse and, in fact, it implies that $L_{t_{i_{j}}}\left(\partial \tilde{K}_{t_{i_{j}}}\right)$ converges to the unit circle in the Hausdorff metric.

\section{EXPANDING $p$-FLOW}

Lemma 5.1. As $K_{t}$ evolves by the centro-affine curvature flow (1.2), its dual $K_{t}^{\circ}$ evolves under the flow

$$
\frac{\partial}{\partial t} s=s\left(\frac{\kappa}{s^{3}}\right)^{-\frac{p}{p+2}}, s(\cdot, t)=s_{\partial K_{t}^{\circ}}(\cdot), s(\cdot, 0)=s_{\partial K_{0}^{\circ}}(\cdot) .
$$

Proof. The proof of Lemma 5.1] is given in 31, but for completeness we will present it here. Recall that $A\left(K^{\circ}\right)=\frac{1}{2} \int_{\mathbb{S}^{1}} \frac{1}{s^{2}} d \theta$ and that, under sufficient regularity assumptions on $\partial K$ which are satisfied here, $\Omega_{q}(K)=\Omega_{\frac{4}{q}}\left(K^{\circ}\right)$ for any $q \neq-n$, in which case the $q$-affine length is not defined. Therefore, as $K_{t}$ evolves by the centro-affine curvature flow (1.2), the volume of the dual body $K_{t}^{\circ}$ changes by

$$
\frac{d}{d t} A^{\circ}(t)=\Omega_{-\frac{p}{p+1}}^{\circ}(t)
$$

where the notation stands for $\Omega_{-\frac{p}{p+1}}\left(K_{t}^{\circ}\right)$. Compared with the rate of change of the area of a convex body $L$ whose boundary is deformed by a normal vector field with speed $v$, which is $\frac{d}{d t} A(L)=\int_{\mathbb{S}^{1}} v \frac{1}{\kappa_{L}} d \theta$, we infer that while $K_{t}$ evolve, up to diffeomorphisms, by (1.2), their duals $K_{t}^{\circ}$ evolve, up to diffeomorphisms, by (5.1). 
Similar to Propositions 2.1] and 2.2 of [31, we have

Proposition 5.2. Let $K_{0}$ be a convex body belonging to $\mathcal{K}_{\text {sym }}$ and let $p \geq 1$. Then there exists a time $T>0$ for which equation (5.1) has a unique solution starting from $K_{0}$.

Proposition 5.3 (Containment principle). If $K_{\text {in }}$ and $K_{\text {out }}$ are the two convex bodies in $\mathcal{K}_{\text {sym }}$ such that $K_{\text {int }} \subset K_{\text {out }}$, and $p \geq 1$, then $K_{\text {in }}(t) \subseteq K_{\text {out }}(t)$ for as long as the solutions $K_{\text {in }}(t)$ and $K_{\text {out }}(t)$ (with given initial data $K_{\text {in }}(0)=K_{\text {in }}$, $\left.K_{\text {out }}(0)=K_{\text {out }}\right)$ of (5.1) exist in $\mathcal{K}_{\text {sym }}$.

Similar to Lemma 2.3 we have

Lemma 5.4. Let $\left\{K_{t}\right\}_{t}$ be a solution of (5.1) where $K_{0} \in \mathcal{K}_{\text {sym }}$. Then $K_{t} \in \mathcal{K}_{\text {sym }}$ as long as the flow exists.

Combining Proposition 2.10, Lemma 5.1, and Propositions 5.2 and 5.3 we obtain

Proposition 5.5. Suppose $K_{t}$ is a family of convex bodies such that it evolves under the flow

with $p \geq 1$. Then

$$
\frac{\partial}{\partial t} s(\cdot, t)=s\left(\frac{\kappa}{s^{3}}\right)^{-\frac{p}{p+2}}(\cdot, t)
$$

$$
\forall \theta: \quad \lim _{t \rightarrow T} s(\theta, t)=\infty .
$$

Proposition 5.6. Ellipses centered at the origin are the only homothetic solutions to (5.1).

Proof. The proof follows from the duality between the two flows and Proposition 2.11 .

Furthermore, we obtain:

Theorem 5.7. Let $p>1$. Suppose $\tilde{K}_{t}$ is a solution of the normalized flow derived from (5.1) for some initial convex body in $\mathcal{K}_{\text {sym. }}$. Then there exists a family of linear transformations $\left\{L_{t}\right\}_{t \in[0, T)} \subset S L(2)$ such that $L_{t}\left(\partial \tilde{K}_{t}\right)$ converge to $\mathbb{S}^{1}$ in the Hausdorff metric.

Proof. Let $\left\{L_{t}\right\}_{t \in[0, T)}$ be the family of length minimizing special linear transformations that we defined in the proof of Theorem 4.6. Since $\left(L_{t}\left(\tilde{K}_{t}\right)\right)^{\circ}=L_{t}^{-t}\left(\tilde{K}_{t}^{\circ}\right)$, where $L_{t}^{-t}$ is the inverse transpose of $L_{t}$, the claim follows.

\section{A PROOF OF THE $p$-AFFINE ISOPERIMETRIC INEQUALITY}

In this section we aim to provide a new proof of the $p$-affine isoperimetric inequality, $p \geq 1$, for a convex body $K \in \mathcal{K}_{\text {sym }}$. Since our proofs of Theorems 4.6] and 5.7 are dependent on the $p$-affine isoperimetric inequalities, we cannot apply our results on $p$-affine flows to obtain the $p$-affine isoperimetric inequalities. Instead we employ the affine normal flow to reach our goal; see [5].

We state the following general evolution equation for $\Omega_{l}$ under the contracting $p$-affine flow for any $l \in \mathbb{R}$ :

$$
\frac{d}{d t} \Omega_{l}(t)=\frac{2(l-2)}{l+2} \int_{\gamma} \sigma^{1-\frac{3 p}{p+2}-\frac{3 l}{l+2}} d \mathfrak{s}+\frac{18 p l}{(l+2)^{2}(p+2)} \int_{\gamma} \sigma^{-\frac{3 p}{p+2}-\frac{3 l}{l+2}} \sigma_{\mathfrak{s}}^{2} d \mathfrak{s} .
$$

Proof of this equation is parallel to the one in part (4) of Lemma 3.1. 
Lemma 6.1. The following sharp affine isoperimetric inequalities hold along the affine normal flow. If $1 \leq l \leq 2$, then

$$
\frac{d}{d t} \Omega_{l}(t) \geq \frac{l-2}{l+2} \frac{\Omega_{l} \Omega_{1}}{A}+\frac{2(l-1)\left(4 l^{2}+3 l+2\right)}{(l+2)^{3}} \int_{\gamma} \sigma^{-1-\frac{3 l}{l+2}} \sigma_{\mathfrak{s}}^{2} d \mathfrak{s},
$$

while, if $l \geq 2$, we then have

$$
\frac{d}{d t} \Omega_{l}(t) \geq \frac{l-2}{l+2} \frac{\Omega_{l} \Omega_{1}}{A}+\frac{6 l}{(l+2)^{2}} \int_{\gamma} \sigma^{-1-\frac{3 l}{l+2}} \sigma_{\mathfrak{s}}^{2} d \mathfrak{s} .
$$

Proof. Before presenting a proof of the second claim let us to state the following generalized Hölder inequality developed by Andrews [3. If $M$ is a compact manifold with a volume form $d \omega, g$ is a continues function on $M$, and $F$ is a decreasing real, positive function, then

$$
\frac{\int_{M} g F(g) d \omega}{\int_{M} F(g) d \omega} \leq \frac{\int_{M} g d \omega}{\int_{M} d \omega} .
$$

If $F$ is strictly decreasing, then equality occurs if and only if $g$ is constant.

Define $d \omega=\sigma d \mathfrak{s}, g=\sigma$, and $F(x):=x^{-\frac{3 l}{l+2}}$. Furthermore, observe that for a convex body $K$ in $\mathbb{R}^{2}$ we have $2 A=\int_{\partial K} \sigma d \mathfrak{s}$. This implies

$$
\int_{\partial K} \sigma^{-\frac{3 l}{l+2}} d \mathfrak{s} \geq \frac{\Omega_{l} \Omega_{1}}{2 A}
$$

hence the second claim follows by this last inequality and the evolution equation (6.1) for $p=1$. To prove the first inequality, one can proceed similarly as in the proof of inequality (3.7), and use the affine-geometric Wirtinger inequality developed by Andrews [4, Lemma 6].

Lemma 6.2. Let $l \geq 1$. Then the l-affine isoperimetric ratio, $\frac{\Omega_{l}^{2+l}(t)}{A^{2-l}(t)}$, is nondecreasing along the affine normal flow and remains constant if and only if $K_{t}$ is an origin centered ellipse.

Proof.

$$
\begin{aligned}
\frac{d}{d t} \frac{\Omega_{l}^{2+l}(t)}{A^{2-l}(t)} & =\frac{(2+l) \Omega_{l}^{l+1}(t) A^{2-l}(t) \frac{d}{d t} \Omega_{l}+(2-l) A^{1-l}(t) \Omega_{l}^{2+l}(t) \Omega_{p}(t)}{A^{2(2-l)}(t)} \\
& =\frac{\Omega_{l}^{l+1}(t)}{A^{2-l}(t)}\left((2+l) \frac{d}{d t} \Omega_{l}-(l-2) \frac{\Omega_{l}(t) \Omega_{1}(t)}{A(t)}\right) \geq 0,
\end{aligned}
$$

where we used Lemma 6.1 in the last line.

Theorem 6.3. Let $l \geq 1$. Then the following l-affine isoperimetric inequality holds for a convex body $K \in \mathcal{K}_{\text {sym }}$ :

$$
\frac{\Omega_{l}^{2+l}(K)}{A^{2-l}(K)} \leq 2^{l+2} \pi^{2 l} ;
$$

moreover, equality holds if and only if for centered ellipses at origin.

Proof. The claim is an immediate consequence of the weak convergence of the solutions of the normalized affine normal flow to a centered ellipse and Lemma 6.2 . 


\section{ACKNOWLEDGMENTS}

The author would like to immensely thank Alina Stancu for many useful discussions on this work and for her help and advice on various parts of this paper. The author would also like to thank the referee for helpful comments and suggestions.

\section{REFERENCES}

[1] Ben Andrews, Contraction of convex hypersurfaces in Euclidean space, Calc. Var. Partial Differential Equations 2 (1994), no. 2, 151-171, DOI 10.1007/BF01191340. MR1385524 (97b:53012)

[2] Ben Andrews, Contraction of convex hypersurfaces by their affine normal, J. Differential Geom. 43 (1996), no. 2, 207-230. MR.1424425 (97m:58045)

[3] Ben Andrews, Evolving convex curves, Calc. Var. Partial Differential Equations 7 (1998), no. 4, 315-371, DOI 10.1007/s005260050111. MR.1660843 (99k:58038)

[4] Ben Andrews, The affine curve-lengthening flow, J. Reine Angew. Math. 506 (1999), 43-83, DOI 10.1515/crll.1999.008. MR.1665677(2000e:53081)

[5] Ben Andrews, Motion of hypersurfaces by Gauss curvature, Pacific J. Math. 195 (2000), no. 1, 1-34, DOI 10.2140/pjm.2000.195.1. MR1781612(2001i:53108)

[6] Sigurd Angenent, Guillermo Sapiro, and Allen Tannenbaum, On the affine heat equation for non-convex curves, J. Amer. Math. Soc. 11 (1998), no. 3, 601-634, DOI 10.1090/S0894-034798-00262-8. MR.1491538 (99d:58039)

[7] Andrea Cianchi, Erwin Lutwak, Deane Yang, and Gaoyong Zhang, Affine Moser-Trudinger and Morrey-Sobolev inequalities, Calc. Var. Partial Differential Equations 36 (2009), no. 3, 419-436, DOI 10.1007/s00526-009-0235-4. MR2551138(2010h:46041)

[8] S. Ivanov, Private communication.

[9] Fritz John, Extremum problems with inequalities as subsidiary conditions, Studies and Essays Presented to R. Courant on his 60th Birthday, January 8, 1948, Interscience Publishers, Inc., New York, N. Y., 1948, pp. 187-204. MR0030135(10,719b)

[10] N. V. Krylov, Nonlinear elliptic and parabolic equations of the second order, Mathematics and its Applications (Soviet Series), vol. 7, D. Reidel Publishing Co., Dordrecht, 1987. Translated from the Russian by P. L. Buzytsky [P. L. Buzytskiū]. MR901759 (88d:35005)

[11] John Loftin and Mao-Pei Tsui, Ancient solutions of the affine normal flow, J. Differential Geom. 78 (2008), no. 1, 113-162. MR2406266 (2009e:53084)

[12] Monika Ludwig and Matthias Reitzner, A classification of $\operatorname{SL}(n)$ invariant valuations, Ann. of Math. (2) 172 (2010), no. 2, 1219-1267, DOI 10.4007/annals.2010.172.1223. MR2680490 (2011g:52025)

[13] Erwin Lutwak, The Brunn-Minkowski-Firey theory. II. Affine and geominimal surface areas, Adv. Math. 118 (1996), no. 2, 244-294, DOI 10.1006/aima.1996.0022. MR1378681 (97f:52014)

[14] Erwin Lutwak and Vladimir Oliker, On the regularity of solutions to a generalization of the Minkowski problem, J. Differential Geom. 41 (1995), no. 1, 227-246. MR1316557(95m:52007)

[15] Erwin Lutwak, Deane Yang, and Gaoyong Zhang, Optimal Sobolev norms and the $L^{p}$ Minkowski problem, Int. Math. Res. Not., posted on 2006, Art. ID 62987, 21, DOI 10.1155/IMRN/2006/62987. MR2211138(2007d:52007)

[16] Erwin Lutwak, Deane Yang, and Gaoyong Zhang, Cramér-Rao and moment-entropy inequalities for Renyi entropy and generalized Fisher information, IEEE Trans. Inform. Theory 51 (2005), no. 2, 473-478, DOI 10.1109/TIT.2004.840871. MR2236062 (2008a:94056)

[17] Erwin Lutwak, Deane Yang, and Gaoyong Zhang, Sharp affine $L_{p}$ Sobolev inequalities, J. Differential Geom. 62 (2002), no. 1, 17-38. MR1987375 (2004d:46039)

[18] Erwin Lutwak, Deane Yang, and Gaoyong Zhang, The Cramer-Rao inequality for star bodies, Duke Math. J. 112 (2002), no. 1, 59-81, DOI 10.1215/S0012-9074-02-11212-5. MR.1890647 (2003f:52006)

[19] Erwin Lutwak, Deane Yang, and Gaoyong Zhang, $L_{p}$ affine isoperimetric inequalities, J. Differential Geom. 56 (2000), no. 1, 111-132. MR1863023 (2002h:52011)

[20] Katsumi Nomizu and Takeshi Sasaki, Affine differential geometry, Cambridge Tracts in Mathematics, vol. 111, Cambridge University Press, Cambridge, 1994. Geometry of affine immersions. MR 1311248 (96e:53014) 
[21] C. M. Petty, Affine isoperimetric problems, Discrete geometry and convexity (New York, 1982), Ann. New York Acad. Sci., vol. 440, New York Acad. Sci., New York, 1985, pp. 113127, DOI 10.1111/j.1749-6632.1985.tb14545.x. MR809198(87a:52014)

[22] L. A. Santaló, An affine invariant for convex bodies of $n$-dimensional space (Spanish), Portugaliae Math. 8 (1949), 155-161. MR0039293 (12,526f)

[23] Guillermo Sapiro and Allen Tannenbaum, Image smoothing based on an affine invariant flow, in: Proceedings of Conference on Information Sciences and Systems, Johns Hopkins University, March, 1993

[24] Guillermo Sapiro and Allen Tannenbaum, Affine invariant scale-space, Internat. J. Comput. Vision 11, 25-44, 1993.

[25] Guillermo Sapiro and Allen Tannenbaum, On affine plane curve evolution, J. Funct. Anal. 119 (1994), no. 1, 79-120, DOI 10.1006/jfan.1994.1004. MR.1255274 (94m:58049)

[26] Guillermo Sapiro and Allen Tannenbaum, On invariant curve evolution and image analysis, Indiana Univ. Math. J. 42 (1993), no. 3, 985-1009, DOI 10.1512/iumj.1993.42.42046. MR,1254129 (94m:58048)

[27] Rolf Schneider, Convex bodies: The Brunn-Minkowski theory, Encyclopedia of Mathematics and its Applications, vol. 44, Cambridge University Press, Cambridge, 1993. MR 1216521 (94d:52007)

[28] Carsten Schütt and Elisabeth Werner, The convex floating body, Math. Scand. 66 (1990), no. 2, 275-290. MR1075144 (91i:52005)

[29] Alina Stancu, The floating body problem, Bull. London Math. Soc. 38 (2006), no. 5, 839-846, DOI 10.1112/S0024609306018728. MR2268369 (2007k:52011)

[30] Alina Stancu, Two volume product inequalities and their applications, Canad. Math. Bull. 52 (2009), no. 3, 464-472, DOI 10.4153/CMB-2009-049-0. MR2547812(2011b:52011)

[31] Alina Stancu, Centro-affine invariants for smooth convex bodies, Int. Math. Res. Not. IMRN 10 (2012), 2289-2320, DOI 10.1093/imrn/rnr110. MR2923167

[32] Neil S. Trudinger and Xu-Jia Wang, The Bernstein problem for affine maximal hypersurfaces, Invent. Math. 140 (2000), no. 2, 399-422, DOI 10.1007/s002220000059. MR1757001 (2001h:53016)

[33] Neil S. Trudinger and Xu-Jia Wang, Boundary regularity for the Monge-Ampère and affine maximal surface equations, Ann. of Math. (2) 167 (2008), no. 3, 993-1028, DOI 10.4007/annals.2008.167.993. MR2415390 (2010h:35168)

[34] Kaising Tso, Deforming a hypersurface by its Gauss-Kronecker curvature, Comm. Pure Appl. Math. 38 (1985), no. 6, 867-882, DOI 10.1002/cpa.3160380615. MR812353(87e:53009)

Department of Mathematics and Statistics, Concordia University, Montreal, QueBEC, CANADA H3G $1 \mathrm{M} 8$

E-mail address: mivaki@mathstat.concordia.ca 